\title{
Avaliação do cálculo da iluminação natural realizada pelo programa EnergyPlus
}

\author{
Evaluation of daylight calculated by the Energyplus \\ program
}

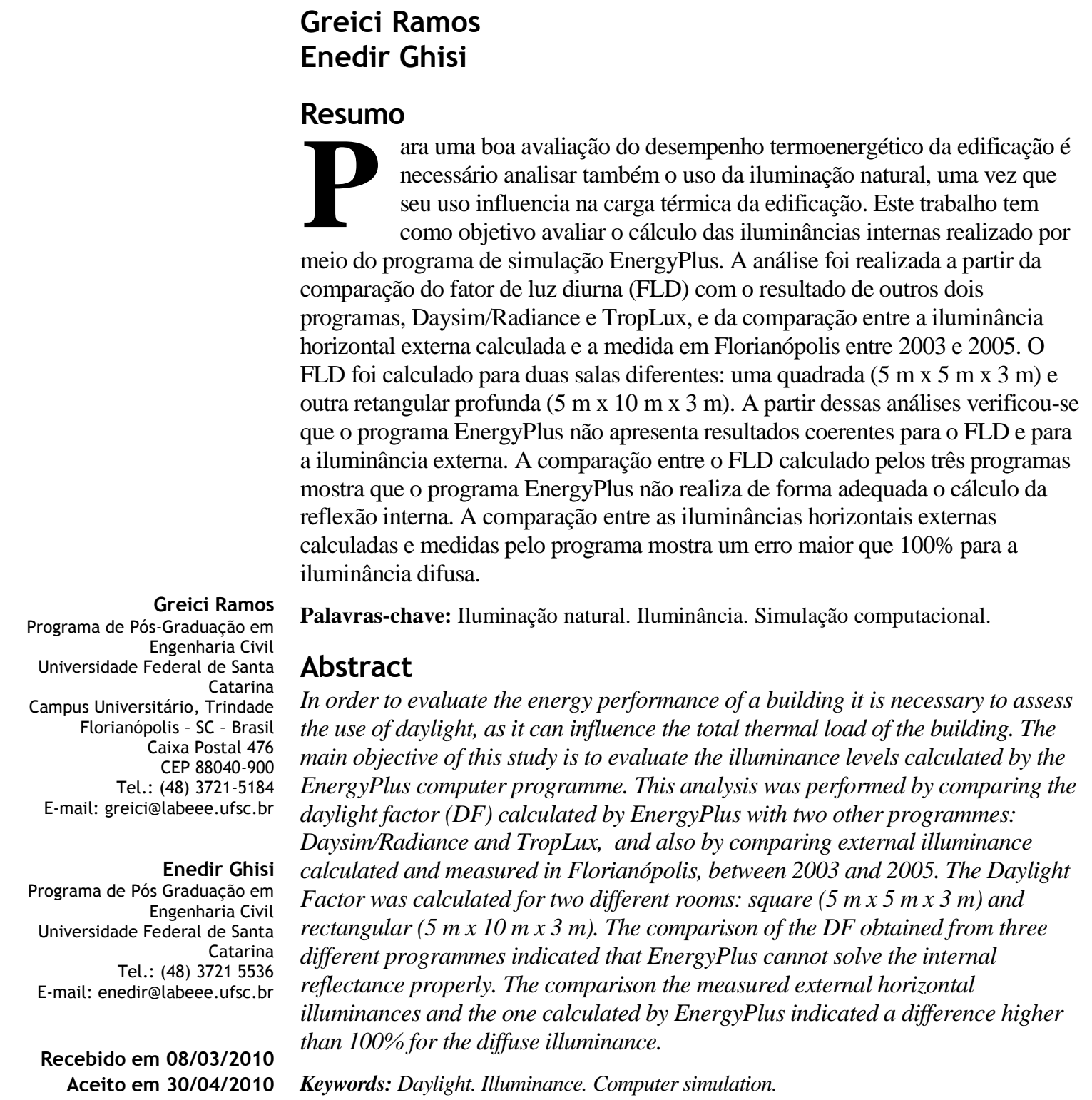




\section{Introdução}

A iluminação natural é uma importante estratégia para uma arquitetura mais eficiente e integrada ao clima onde está inserida. Para tanto, é necessário o estudo da luz natural no ambiente, assim como as trocas de calor que ocorrem através da abertura. $\mathrm{O}$ programa de simulação EnergyPlus possibilita a análise conjunta das trocas de calor e do ganho da iluminação natural pela abertura, onde o cálculo da iluminação natural é realizado com o fator de luz diurna e da iluminação externa, que, por sua vez, é calculada pelo modelo desenvolvido por Perez et al. (1990).

O uso de programas simuladores facilita a análise de grande quantidade de dados, necessários para o cálculo do desempenho termoenergético e luminoso de edificações. A partir dos resultados das simulações computacionais e das análises realizadas através destes é possível obter orientações para as decisões de projeto. Assim, neste trabalho são utilizados três programas simuladores: EnergyPlus, Daysim/Radiance e TropLux.

O programa EnergyPlus foi criado a partir da junção de dois programas, BLAST e DOE-2, e trabalha com o balanço de calor do BLAST, com modelo de ar condicionado genérico, novos algoritmos de transferência de calor e fluxo de ar entre zonas (CRAWLEY et al., 1999), enquanto o cálculo da iluminação natural deriva do programa DOE-2 (ENERGYPLUS, 2007).

O cálculo da iluminância interna é feito mediante a integração entre o fator de luz diurna (FLD) referente à parcela da abóbada celeste e o fator de luz do sol (FLS), multiplicados por sua correspondente iluminância externa. Para o cálculo da parcela da iluminação difusa, realiza-se a ponderação do FLD entre dois tipos de céu. O cálculo da iluminância horizontal externa é realizado a partir do modelo de Perez et al. (1990).

Segundo Winkelmann e Selkowitz (1985), a validação da iluminação realizada para o programa EnergyPlus é a mesma realizada para o DOE-2, uma vez que este foi utilizado como base do algoritmo para o EnergyPlus. A validação foi em parte executada mediante a comparação entre os resultados do DOE-2, Superlite e medições no céu artificial do LBL (Lawrence Berkeley Laboratory). Nessas comparações verificou-se uma diferença média de $15 \%$ entre os três métodos, com exceção das áreas muito próximas ou afastadas da janela, onde o método do fluxo dividido (usado para resolver as reflexões internas) superestima a reflexão interna.

O trabalho de Loutzenhiser, Maxell e Manz (2007) mostra a comparação entre os resultados das simulações realizadas pelos programas EnergyPlus e DOE-2 com os de um modelo real. A comparação foi realizada através de modelos de edifícios com uso de sistemas de sombreamentos nas aberturas. Nessa análise, os resultados de um modelo real são comparados aos resultados das simulações.

A Figura 1 mostra a comparação entre os resultados do cálculo das iluminâncias para a fachada sul, que possui abertura com sistemas de vidro com 25,2 $\mathrm{mm}$ (composto de dois vidros claros de $6 \mathrm{~mm}$ e espaço de ar de 13,2 $\mathrm{mm}$ ), com cortinas translúcidas. Segundo os autores, nenhum dos programas apresentou os resultados das iluminâncias, nos pontos de referência, com $95 \%$ de confiança. Nota-se, por meio dessa figura, que o programa EnergyPlus apresenta grande diferença nos valores das iluminâncias quando comparado ao modelo experimental.

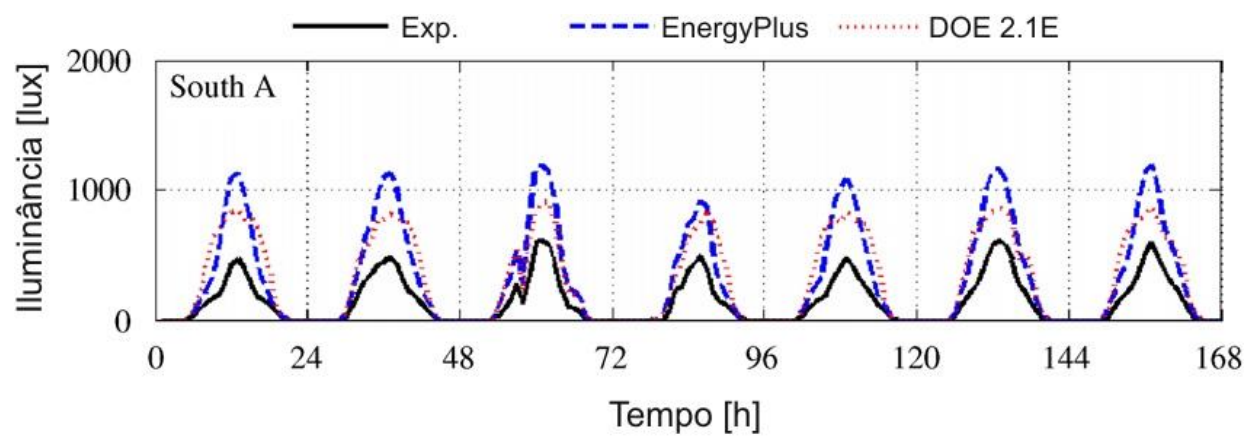

Fonte: Loutzenhiser, Maxell e Manz (2007).

Figura 1 - Iluminância no ponto de referência para a fachada sul, para o modelo experimental e para os modelos simulados nos programas EnergyPlus e DOE-2 
Ramos e Ghisi (2008) mostram uma comparação entre as iluminâncias difusas resultantes de três programas de simulação, EnergyPlus, Daysim/Radiance e TropLux, para uma sala quadrada e uma retangular profunda, simulados para o clima de Florianópolis. Para os programas EnergyPlus e Daysim/Radiance, utilizou-se o arquivo climático (TRY) de Florianópolis, enquanto, para o TropLux, utilizou-se o modelo de Treguenza. Nesse trabalho, os autores mostram uma diferença superior a $50 \%$ entre os programas que utilizam o arquivo climático (EnergyPlus e Daysim/Radiance) e o modelo de cálculo estático (TropLux).

O programa Daysim/Radiance, desenvolvido pelo National Research Council Canada (NRCC) e pelo Fraunhofer Institute for Solar Energy Systems, na Alemanha, foi elaborado com o intuito de calcular as iluminâncias para o período de um ano, de forma rápida e independente do tipo de céu (REINHART, 2006). Esse programa simula a iluminação natural através do coeficiente da luz natural, baseando-se no programa Radiance, que utiliza o método do raio traçado, e no modelo de céu desenvolvido por Perez et al. (1990), para, dessa forma, possibilitar a simulação das iluminâncias sob qualquer condição de céu (REINHART; WALKENHORST, 2001). A simulação é realizada a partir do arquivo climático e de um modelo tridimensional do ambiente a ser analisado, onde são definidas as propriedades ópticas das superfícies.

O programa TropLux é baseado em três conceitos fundamentais: o método Monte Carlo, o método do raio traçado e o conceito de coeficientes de luz natural. O método Monte Carlo trata da abordagem estatística para solucionar integrais múltiplas; a técnica do raio traçado segue o caminho de um raio entre superfícies, cuja principal vantagem consiste na possibilidade de dar soluções teóricas simples para geometrias complexas; e os coeficientes de luz natural relacionam a iluminância em determinada superfície, a partir de dada subdivisão do céu, e a iluminância normal em um plano desobstruído, a partir dessa mesma subdivisão. Para o cálculo do coeficiente da luz natural, o programa TropLux adota dois tipos de subdivisões do céu: para a componente refletida, utiliza a subdivisão proposta pela CIE (Commission Internationale de l'Eclairage) de 145 partes; e para o cálculo da componente direta, utiliza uma subdivisão maior, 5.221 partes, a fim de melhorar a precisão dos resultados em função do tamanho angular do sol (CABÚS, 2005). Para a realização da simulação da distribuição da luz natural em um ambiente, ainda são necessários alguns dados de entrada, como geometria da sala, planos, janelas e características dos materiais, além da localização geográfica. Esse programa simula as iluminâncias a partir de três bases de cálculo: modelo de Tregenza, da Illuminating Engineering Society (IES) e a partir de dados locais.

Dadas as diferenças encontradas entre as iluminâncias resultantes da simulação realizada no programa EnergyPlus, este artigo visa complementar o trabalho de Ramos e Ghisi (2008), a partir de uma análise mais detalhada do método de cálculo utilizado pelo EnergyPlus.

Este artigo tem o objetivo de avaliar o cálculo da iluminação natural efetuado pelo programa EnergyPlus, através da comparação do fator de luz diurna e da comparação das iluminâncias externas resultantes da simulação com dados medidos na cidade de Florianópolis.

\section{Método de pesquisa}

As simulações foram realizadas para dois modelos diferentes, sendo estes uma sala quadrada, na proporção de $1: 1(5 \mathrm{~m}$ x $5 \mathrm{~m}$ x $3 \mathrm{~m})$, e uma sala retangular profunda, com proporção de 1:2 (5 m x $10 \mathrm{~m} \times 3 \mathrm{~m})$, representadas na Figura 2. Esses modelos possuem percentual de janela na fachada (PJF) de 50\%, com peitoril de 1,5 m e largura igual à da sala; a abertura é voltada para a fachada sul. Nas aberturas considerou-se o uso de vidro claro de $3 \mathrm{~mm}$, com transmissividade à luz visível igual a 0,88 . Os modelos possuem paredes e teto brancos, com refletância igual a 0,85 e piso bege, com refletância igual a 0,60. Em todas as simulações considerou-se a superfície de trabalho a $0,75 \mathrm{~m}$ do piso.

Os dados utilizados para as análises foram coletados pelo LabSolar, enquanto as iluminâncias, utilizadas para as simulações no programa TropLux, foram coletadas pela Estação de Medição de Iluminação Natural de Florianópolis (EMIN-Floripa), através do Laboratório de Energia Solar (LabSolar) e do Laboratório de Conforto Ambiental (LabCon), ambos da UFSC. Os dados coletados serão utilizados em base horária.

\section{Verificação do cálculo do fator de luz diurna}

Sabendo que o cálculo da iluminação interna no programa EnergyPlus é realizado com base no fator de luz diurna (FLD), optou-se por comparar os valores desse índice com os dos outros dois programas.

As simulações foram realizadas para os dois modelos. Em cada um deles verificou-se o FLD de cinco pontos ao longo da sala, todos alinhados com 
o meio da janela, a uma altura de $0,75 \mathrm{~m}$, de acordo com a Figura 3. Como mencionado anteriormente, o programa EnergyPlus realiza o cálculo de apenas dois pontos de referência, a partir dos quais calcula a iluminância, e pondera esses valores para os outros pontos desejados. Essa verificação foi realizada para cinco pontos, para possibilitar a análise da resposta do programa à medida que aumenta a distância entre o ponto analisado e a abertura.

As simulações foram realizadas a partir do arquivo climático, com base nos dados de 2005, e latitude de Florianópolis.

Os programas Daysim/Radiance e TropLux fornecem o FLD como resultado da simulação. Já para o programa EnergyPlus o FLD é calculado a partir dos dados de um dia de céu encoberto, a partir da Equação 1.

$\mathrm{FLD}=\left(\mathrm{E}_{\mathrm{p}} / \mathrm{E}_{\mathrm{h} \mathrm{ext}}\right) \times 100 \%$

Eq. 1

Onde:

FLD: fator de luz diurna [\%];

$\mathrm{E}_{\mathrm{p}}$ : iluminância difusa no ponto de referência [1x];

$\mathrm{E}_{\mathrm{h} \text { ext }}$ : iluminância difusa horizontal externa [1x].

\section{Avaliação do modelo desenvolvido por Perez}

Nesta etapa foram comparadas as iluminâncias difusa e direta horizontal externa, coletadas pela estação EMIN-Floripa, com as utilizadas pelo programa EnergyPlus para o cálculo da iluminância interna. Esta é obtida pelo método desenvolvido por Perez et al. (1990). Assim, para essa comparação foram utilizados dados de radiação, temperatura, ângulo zenital e umidade, medidos em Florianópolis, de 2003 a 2005, pela estação de medição do LabSolar, localizada em Florianópolis; e iluminâncias, medidas pela EMIN-Floripa, através do LabSolar e do LabCon.

O modelo de Perez et al. (1990) baseia-se em quatro parâmetros, para caracterizar o céu e estimar a iluminância, sendo eles: índice de claridade do céu ( $\varepsilon-$ da Equação 2); ângulo zenital (Z); índice do brilho do céu ( $\Delta$ - Equação 3); e vapor de água na atmosfera (W -Equação 4). Esses parâmetros foram calculados a partir dos dados coletados pelo LabSolar a partir das equações a seguir.

$\varepsilon=\frac{\left[\frac{\left(I_{D h}+I\right)}{I_{D h}}+k Z^{3}\right]}{\left(1+k Z^{3}\right)}$

Eq. 2
Onde:

$\varepsilon:$ índice de claridade do céu, adimensional;

IDh: radiação difusa horizontal $\left[\mathrm{W} / \mathrm{m}^{2}\right]$;

I: radiação normal direta $\left[\mathrm{W} / \mathrm{m}^{2}\right]$;

$\mathrm{K}$ : constante igual a 1,041 ; e

Z: ângulo zenital [rad].

$\Delta=\left(\mathrm{I}_{\mathrm{Dh}} \times \mathrm{m}\right) / \mathrm{I}_{0}$

Onde:

$\Delta$ : índice do brilho do céu, adimensional;

$\mathrm{I}_{\mathrm{Dh}}$ : radiação difusa horizontal $\left[\mathrm{W} / \mathrm{m}^{2}\right]$;

m: massa de ar óptica relativa, que pode ser determinada por: $\mathrm{m}=1 / \mathrm{sen} \gamma_{\mathrm{s}}$;

$\gamma_{\mathrm{s}}:$ altitude solar [rad]; e

$\mathrm{I}_{0}$ : radiação extraterrestre $\left[\mathrm{W} / \mathrm{m}^{2}\right]$.

$\mathrm{W}=\exp (0,07 \times \mathrm{Td}-0,075)$

Eq. 4

Onde:

$\mathrm{W}$ : vapor de água na atmosfera $[\mathrm{cm}]$; e

Td: temperatura de orvalho $\left[{ }^{\circ} \mathrm{C}\right]$.

A iluminância horizontal difusa foi calculada a partir da Equação 5, e a iluminância direta, a partir da Equação 6. Nessas equações utilizam-se diferentes coeficientes, de acordo com cada categoria da claridade do céu, conforme a Tabela 1 .

$E_{\mathrm{hDif}}=\mathrm{I}_{\mathrm{Dh}}\left[a_{i}+b_{i} \mathrm{~W}+c_{i} \cos (\mathrm{Z})+d_{i}\right.$ In $\left.\Delta\right] \quad$ Eq. 5

$\mathrm{E}_{\mathrm{hDir}}=\operatorname{máx}\left\{0,1\left[a_{i}+b_{i} \mathrm{~W}+\right.\right.$

$\left.\left.c_{i} \exp (5,73 Z-5)+d_{i} \Delta\right]\right\}$

Eq. 6

Onde:

$\mathrm{E}_{\mathrm{hDif}}$ : a iluminância horizontal difusa [1x];

$\mathrm{E}_{\mathrm{hDir}}$ : iluminância horizontal direta $[\mathrm{lx}]$;

$\mathrm{I}_{\mathrm{Dh}}$ : radiação difusa horizontal $\left[\mathrm{W} / \mathrm{m}^{2}\right]$;

I: radiação direta $\left[\mathrm{W} / \mathrm{m}^{2}\right]$;

$\mathrm{W}$ : vapor de água na atmosfera $[\mathrm{cm}]$;

Z: ângulo zenital [rad]; e

$\Delta:$ índice do brilho do céu;

$a_{i}, b_{i}, c_{i}, d_{i}:$ coeficientes obtidos através da Equação 2, de acordo com o índice de claridade do céu $(\varepsilon)$. 


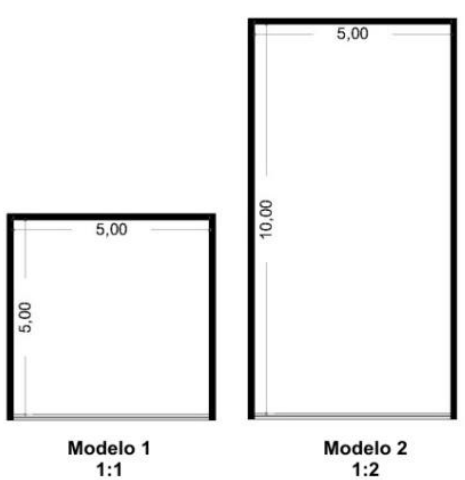

Figura 2 - Dimensões dos modelos estudados

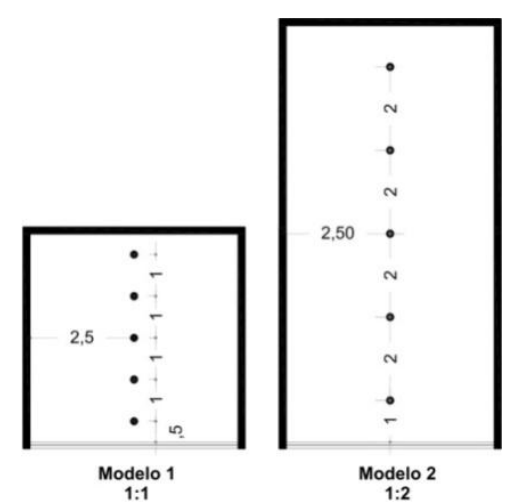

Figura 3 - Localização dos pontos nas salas para análise do fator de luz diurna

\begin{tabular}{l|c|c|c|c|c|c|c|c}
\hline & \multicolumn{4}{|c|}{ Iluminância Horizontal Difusa } & \multicolumn{4}{c}{ Iluminância Horizontal Direta } \\
\cline { 2 - 9 } & $\mathbf{a}_{\mathbf{i}}$ & $\mathbf{b}_{\mathbf{i}}$ & $\mathbf{c}_{\mathbf{i}}$ & $\mathbf{d}_{\mathbf{i}}$ & $\mathbf{a}_{\mathbf{i}}$ & $\mathbf{b}_{\mathbf{i}}$ & $\mathbf{c}_{\mathbf{i}}$ & $\mathbf{d}_{\mathbf{i}}$ \\
\hline 1 & 97,24 & $-0,46$ & 12,00 & $-8,91$ & 57,20 & $-4,55$ & $-2,98$ & 117,12 \\
\hline 2 & 107,22 & 1,15 & 0,59 & $-3,95$ & 98,99 & $-3,46$ & $-1,21$ & 12,38 \\
\hline 3 & 104,97 & 2,96 & $-5,53$ & $-8,77$ & 109,83 & $-4,90$ & $-1,71$ & $-8,81$ \\
\hline 4 & 102,39 & 5,59 & $-13,95$ & $-13,90$ & 110,34 & $-5,84$ & $-1,99$ & $-4,56$ \\
\hline 5 & 100,71 & 5,94 & $-22,75$ & $-23,74$ & 106,36 & $-3,97$ & $-1,75$ & $-6,16$ \\
\hline 6 & 106,42 & 3,83 & $-36,15$ & $-28,83$ & 107,19 & $-1,25$ & $-1,51$ & $-26,73$ \\
\hline 7 & 141,88 & 1,90 & $-53,24$ & $-14,03$ & 105,75 & 0,77 & $-1,26$ & $-34,44$ \\
\hline 8 & 152,23 & 0,35 & $-45,27$ & $-7,98$ & 101,18 & 1,58 & $-1,10$ & $-8,29$ \\
\hline
\end{tabular}

Fonte: Perez et al. (1990).

Tabela 1 - Coeficientes para cálculo das iluminâncias externas

A partir dos dados calculados, verificaram-se as correlações entre as iluminâncias coletadas em Florianópolis e as calculadas por esse modelo. Além disso, para uma melhor avaliação do modelo, foram utilizados os seguintes índices: viés médio (viesM) e o erro quadrático médio (EQM), mostrados nas Equações 7 e 8 respectivamente. A partir do viés é possível verificar a tendência do modelo em superestimar ou subestimar as iluminâncias calculadas, enquanto $\mathrm{o}$ erro quadrático médio mostra o erro absoluto entre as grandezas analisadas.

vies $M=\frac{\sum_{i}^{N}\left(y_{i}-x_{i}\right) / x_{i}}{N}$

$\mathrm{EQM}=\sqrt{\frac{\sum_{\mathrm{i}}^{\mathrm{N}}\left(\left(y_{i}-x_{i}\right) / x_{i}\right)^{2}}{N}}$

Onde:

viesM: viés médio [\%];

EQM: erro quadrático médio [\%];

$\mathrm{y}_{\mathrm{i}}$ : iluminância estimada [1x];

$\mathrm{x}_{\mathrm{i}}$ : iluminância medida [lx]; e

$\mathrm{N}$ : número de valores analisados, adimensional.
Para os modelos de maior e menor EQM realizouse também a análise dos gráficos dos resíduos (diferença entre o valor estimado e o real). Essa análise de modelos de regressão pode indicar ajustes do modelo diante das variáveis independentes e do valor estimado. Dessa forma, serão analisados os gráficos dos resíduos em função do valor estimado (iluminância) e em função das variáveis do modelo.

Segundo Johnson e Wichern (1998), os gráficos dos resíduos em função do valor estimado podem resultar em três distribuições de pontos: quando o gráfico se assemelha à Figura $4 \mathrm{a}$, indica modelos em que a constante da equação foi omitida; gráficos em que a variação não é constante, como na Figura 4b, em que os resíduos formam um padrão de forma afunilada, sugerem a necessidade de modificações na equação; enquanto a distribuição homogênea de pontos, como na Figura $4 c$, mostra que os resíduos não têm dependência do valor estimado, indicando um modelo de regressão coerente. $\mathrm{Na}$ análise dos resíduos em função das variáveis do modelo, a distribuição de pontos em gráficos com padrões sistemáticos, como o encontrado na Figura 4d, indica a necessidade da inclusão de um ou mais termos no modelo. 


\section{Análise de resultados}

A seguir são apresentadas as comparações entre o FLD resultante dos três programas de simulação, EnergyPlus, Daysim/Radiance e TropLux, e a comparação entre as iluminâncias externas para a cidade de Florianópolis.

\section{Avaliação do cálculo do fator de luz diurna}

Como mencionado anteriormente, o programa EnergyPlus realiza o cálculo da iluminância interna através da iluminância externa e do FLD. Assim, verificou-se este índice para as duas geometrias de sala, de acordo com os modelos apresentados na Figura 2.

A Figura 5 foi obtida por meio de simulações para o modelo de proporção $1: 1$, com PJF de $50 \%$. Pode-se verificar um comportamento semelhante entre o FLD dos diferentes programas. Na Figura 5 a o programa Daysim/Radiance tem o FLD máximo próximo ao local de entrada da luz, enquanto para os outros dois programas o FLD máximo está a $1,5 \mathrm{~m}$ da abertura. A Figura $5 \mathrm{~b}$ mostra a diferença absoluta encontrada entre os programas, em que se percebe que, à medida que

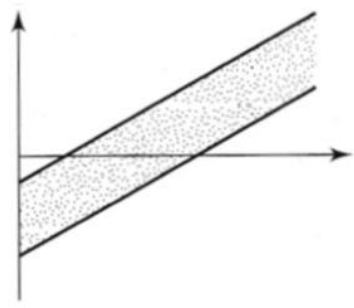

(a)

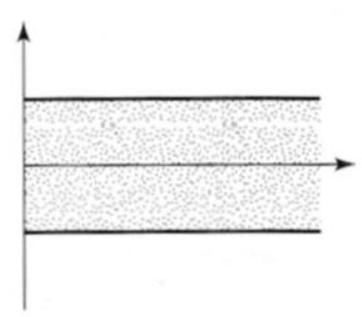

(c) se afasta da abertura, diminui a diferença entre o FLD.

O modelo de proporção 1:2 (5 m x $10 \mathrm{~m}$ x $3 \mathrm{~m})$, representado na Figura 6, possui os menores valores para o FLD. Para esse modelo, o FLD resultante do programa EnergyPlus, após os $6 \mathrm{~m}$, mantém-se praticamente constante, mostrando a baixa sensibilidade do programa para simular a iluminação em locais afastados do ponto de entrada da luz no ambiente. Através dessas imagens pode-se verificar ainda que, enquanto próximo à abertura, o maior FLD é encontrado pelo Daysim/Radiance, e o menor, pelo EnergyPlus. Afastado dela, dá-se o contrário, com o maior FLD para o EnergyPlus, e o menor, para o Daysim/Radiance e o TropLux.

A Figura $6 \mathrm{~b}$ mostra as diferenças entre os resultados encontrados pelos programas para esse modelo, em que se percebe a grande diferença entre os programas em quase toda a sala. Os programas que apresentam maior erro são o EnergyPlus e o Daysim/Radiance, com uma diferença de $10 \%$ entre o FLD no ponto mais próximo à abertura.

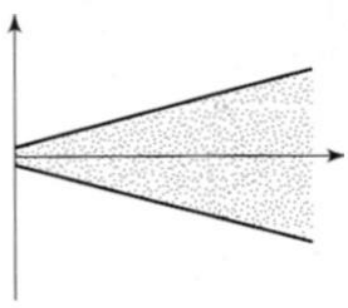

(b)

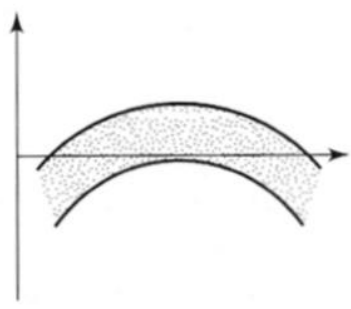

(d)

Fonte: Johson e Wichern (1998).

Figura 4 - Modelos dos gráficos dos resíduos em função dos valores estimados e das variáveis de um modelo de regressão múltipla 


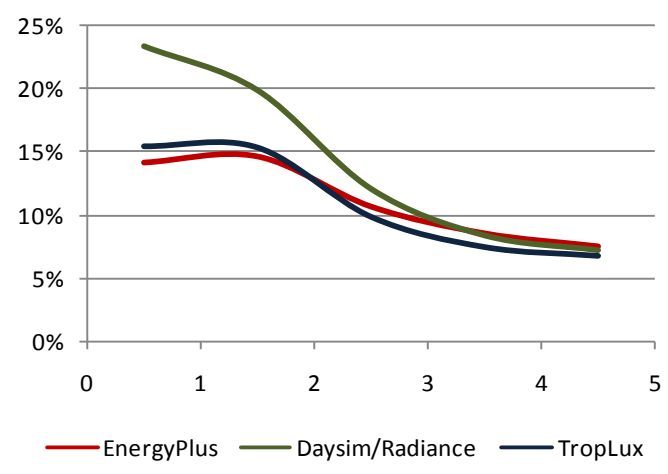

(a) Fator de luz diurna

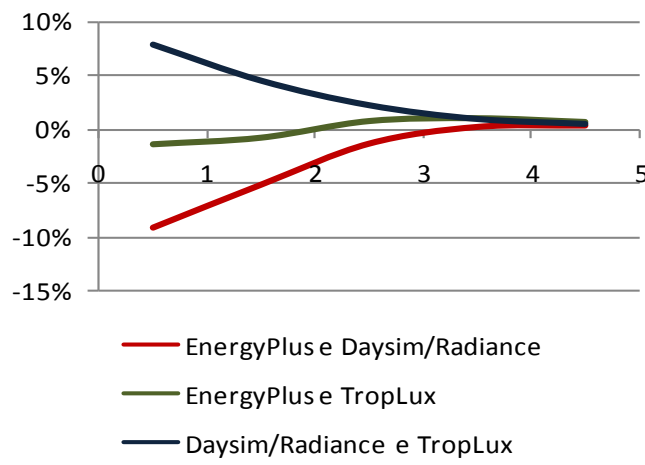

(b) Diferença absoluta encontrada entre os programas

Figura 5 - Fator de luz diurna (FLD) para sala com proporção 1:1, com PJF de 50\%

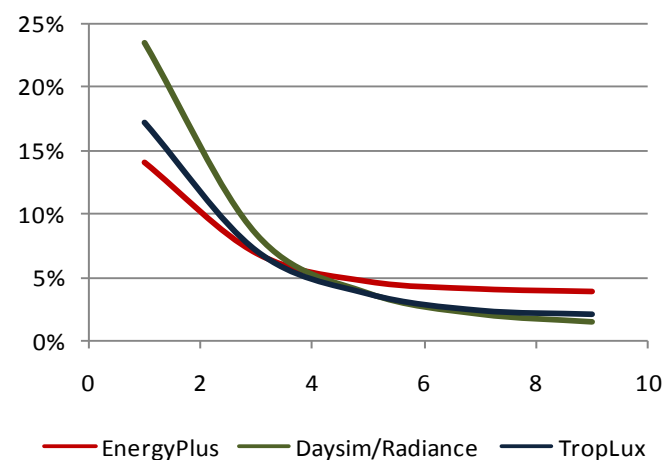

(a) PJF 50\%

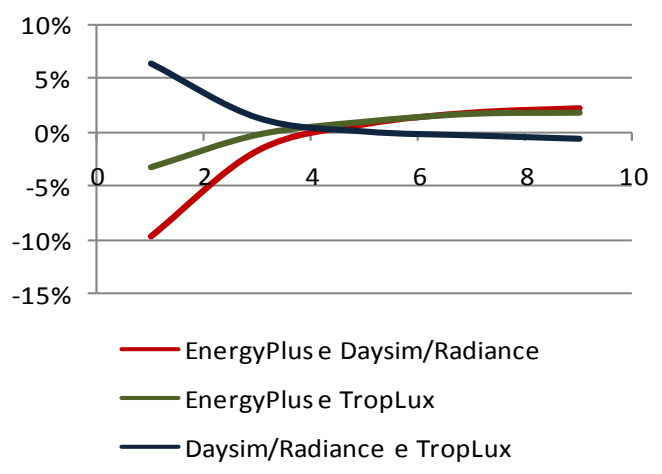

(b) Diferença absoluta encontrada entre os programas

Figura 6 - Fator de luz diurna (FLD) para sala com proporção 1:2, com PJF de 50\%

Com o modelo com proporção de 1:2, verifica-se que, à medida que aumenta a importância da parcela refletida internamente, como no fundo da sala, onde existe menor incidência da luz direta, a diferença entre os resultados do programa EnergyPlus e dos outros dois programas aumenta, enquanto as diferenças entre o Daysim/Radiance e o TropLux diminuem. Uma possível causa para esse aumento na diferença está nos métodos utilizados para resolver a reflexão interna, uma vez que o primeiro programa utiliza o método do fluxo dividido, e o último, o método do raio traçado. Como no método do fluxo dividido a parcela refletida é dividida igualmente pelo espaço, alguns pontos acabam recebendo uma parcela maior do que a que realmente ocorre. Segundo Winkelmann e Selkowitz (1985), salas com formas que se aproximam à cúbica têm melhores resultados para o método do fluxo dividido, utilizado pelo programa EnergyPlus, para resolver a reflexão interna. A partir das comparações acima, tem-se que o modelo que se afasta dessa forma apresenta maior diferença entre os resultados dos programas.

\section{Avaliação do cálculo da iluminância externa}

Para esta avaliação, as iluminâncias horizontais externas foram comparadas com as calculadas através do método desenvolvido por Perez et al. (1990), utilizado pelo programa EnergyPlus no cálculo da iluminância externa, com os dados coletados de 2003 a 2005.

\section{Iluminância externa difusa}

O método desenvolvido por Perez et al. (1990) separa os tipos de céu em oito classes, e para cada uma delas a equação do cálculo da iluminância apresenta diferentes coeficientes. Assim, os resultados apresentados na Figura 7 mostram os dados para todas as classificações de céu.

A correlação entre a iluminância medida e a calculada está demonstrada na Figura 7, em que se percebe a alta dispersão dos pontos, principalmente nos gráficos que representam céus mais encobertos. A Figura 7a mostra a correlação com todos os dados analisados, em que fica claro que os resultados obtidos pelo modelo de Perez et 
al. (1990) são superiores às iluminâncias medidas, uma vez que a linha de tendência não coincide com a de $45^{\circ}$ e se aproxima do eixo relacionado às iluminâncias calculadas.

Para uma melhor análise, os resultados foram separados de acordo com a classificação de Perez. Assim, a Figura 7b está relacionada aos dados de céu encoberto $(1<\varepsilon \leq 1,065)$, e a Figura $7 \mathrm{i}$, aos dados de céu claro $(\varepsilon>6,2)$. Nesses gráficos observa-se que, para as condições de céu encoberto, a dispersão dos pontos é maior que a encontrada nos outros gráficos e que a linha de tendência da correlação se afasta da ideal. A Figura $7 \mathrm{~b}$ e $7 \mathrm{c}$ correlaciona as iluminâncias para o céu encoberto. Nesses gráficos, encontra-se a maior dispersão dos pontos, principalmente para a
Figura $7 \mathrm{~b}$, onde está a maior nuvem de pontos, mostrando a baixa relação entre as duas grandezas nessa classe de céu.

A Tabela 2 mostra o viés e o erro quadrático médio (EQM) entre as iluminâncias medidas e as calculadas. A partir da Tabela 2 pode-se verificar que o modelo de Perez et al. (1990) superestima a iluminância difusa em todas as classes de céu; entre estas o maior viés ocorre para o céu encoberto, seguido do céu intermediário. Confirmando o que foi mostrado na Figura 7, o maior EQM encontrado ocorre para as parcelas de céu encoberto, com um erro superior a $100 \%$, enquanto o céu claro apresenta a melhor resposta do modelo, mas longe do ideal, com um erro de $34 \%$.

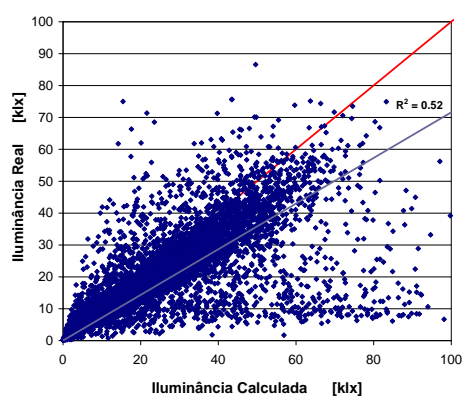

(a) Todos os dados

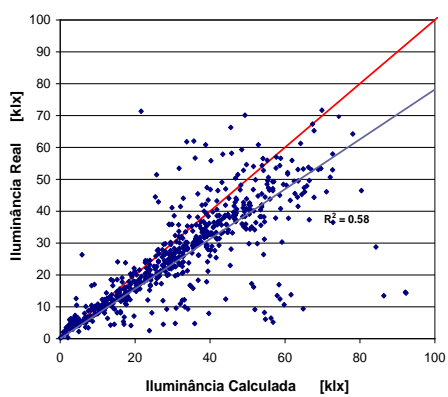

(d) $1,23<\varepsilon \leq 1,5$

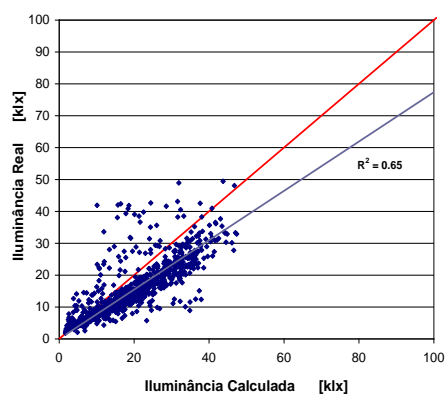

(g) $2,8<\varepsilon \leq 4,5$

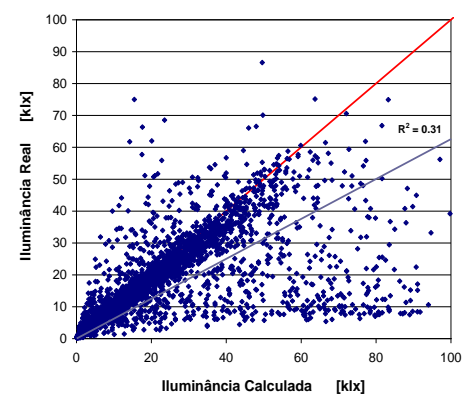

(b) Céu encoberto $-1<\varepsilon \leq 1,065$

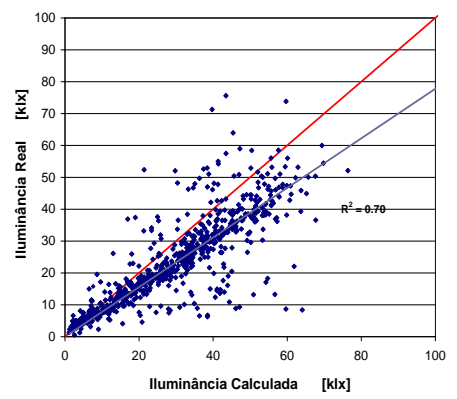

(e) $1,5<\varepsilon \leq 1,95$

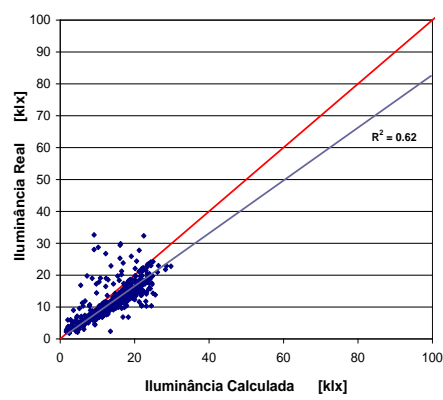

(h) $4,5<\varepsilon \leq 6,2$

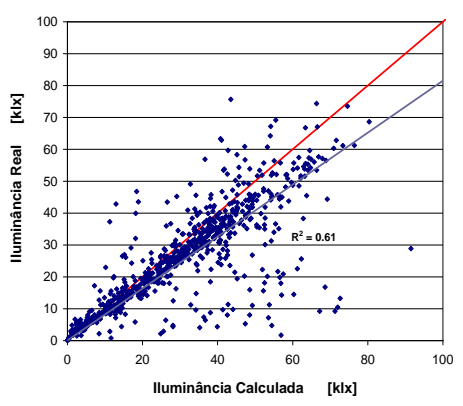

(c) $1,065<\varepsilon \leq 1,23$

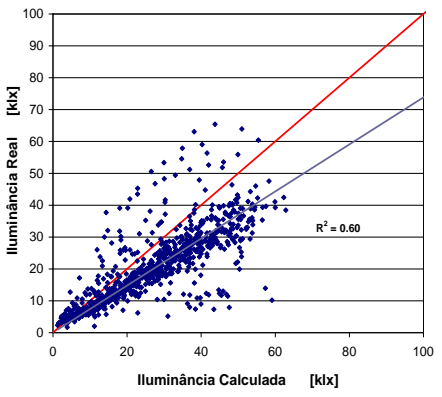

(f) $1,95<\varepsilon \leq 2,8$

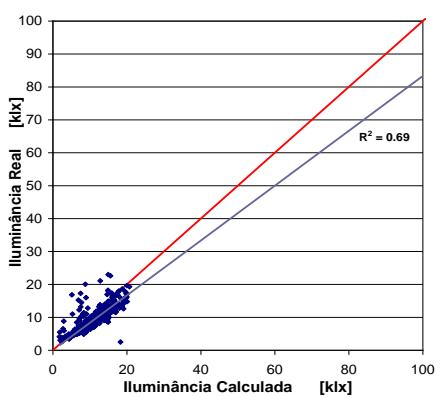

(i) Céu claro - $\varepsilon>6,2$

Figura 7 - Correlação entre as iluminâncias medidas e as calculadas pelo modelo de Perez et al.(1990), através de dados coletados em Florianópolis, entre 2003 e 2005 


\begin{tabular}{l|c|c|c}
\hline & $\begin{array}{c}\text { Iluminância média } \\
\text { medida [klx] }\end{array}$ & Viés [\%] & EQM [\%] \\
\hline Todos & 17,52 & 35,49 & 120,75 \\
\hline 1 & 16,14 & 39,76 & 148,10 \\
\hline 2 & 26,03 & 34,50 & 128,60 \\
\hline 3 & 25,95 & 38,55 & 114,62 \\
\hline 4 & 23,31 & 35,60 & 78,98 \\
\hline 5 & 20,72 & 37,96 & 72,97 \\
\hline 6 & 15,14 & 29,61 & 49,78 \\
\hline 7 & 11,18 & 22,22 & 39,99 \\
\hline 8 & 9,20 & 21,24 & 34,07 \\
\hline
\end{tabular}

Tabela 2 - Iluminância difusa média medida e avaliação estatística do modelo de Perez, para cada classe de céu, a partir de dados de Florianópolis de 2003 a 2005

Em razão do grande erro encontrado entre as iluminâncias horizontais externas obtidas com o modelo de Perez et al. (1990) e as iluminâncias medidas, realizou-se a análise do modelo a partir dos gráficos de resíduos em função das iluminâncias e das variáveis do modelo. Essa análise foi realizada para as classes de céu com maior e menor EQM, céu encoberto e céu claro. A Figura 8 mostra a análise para as iluminâncias medidas e brilho do céu para os dados de céu encoberto, entre 1 e 1,065, faixa que apresenta o maior EQM, enquanto a Figura refere-se aos dados que apresentaram o menor EQM, ou seja, céu claro $\operatorname{com} \varepsilon$ maior que 6,2 .

A Figura 8a mostra os resíduos com forma afunilada, onde, quanto maior o valor previsto, maior o resíduo, indicando a necessidade de modificações na equação. Através da Figura $8 b$ observa-se a ocorrência de um padrão na disposição dos resíduos, o que mostra a necessidade da adição de um ou mais termos na equação. Esse fato não ocorre na Figura 9b, o que indica que a necessidade desses termos está ligada às condições de céu encoberto.

\section{Iluminância externa direta}

A partir do mesmo conjunto de dados compararam-se as iluminâncias diretas medidas com as calculadas. Os resultados são apresentados na Figura 10, de acordo com a classe do céu.

A Figura 10a representa a correlação entre a iluminância medida e a calculada com base em todos os dados coletados, a partir da qual se observa a existência de duas nuvens de pontos, uma, com poucos pontos, em que os dados calculados são maiores que os medidos, e a outra apresenta os dados próximos à linha de correlação ideal. Os gráficos seguintes apresentam as iluminâncias separadas por classe de céu. A Figura 10b, 10c e 10d apresenta as maiores diferenças entre os dados e com as iluminâncias medidas maiores que as calculadas.

A partir do céu intermediário, com $\varepsilon>1,95$, a comparação entre as iluminâncias começa a mostrar melhor correlação entre os dados, em que a linha de tendência está mais próxima da linha de correlação ideal. Dessas classes de céu, a Figura $10 \mathrm{~h}$ e $10 \mathrm{i}$, que representa condições de céu claro, apresenta menor dispersão dos pontos e, consequentemente, menor erro.

As análises do viés e do erro quadrático médio (EQM) entre as iluminâncias medidas e as calculadas são apresentadas na Tabela 10. Verifica-se que o modelo de Perez et al. (1990) tem a tendência de subestimar as iluminâncias para o céu encoberto, com $\varepsilon<1,065$, demonstrado na Figura 10b. A Tabela 3 mostra que o menor EQM encontrado para iluminância direta é de 20,58\%, para a classe de céu 8 , ilustrada na Figura 10 (i).

Para esse modelo também foi realizada a análise do gráfico dos resíduos para as classes de céu com maior e menor EQM. A Figura 11 mostra a análise da eficácia luminosa e do brilho do céu para os dados de céu encoberto $(1,065<\varepsilon \leq 1,23)$, enquanto a Figura refere-se aos dados para o céu claro $(\varepsilon>6,2)$. As Figuras 11a e 12a mostram os resíduos em função da iluminância calculada com forma afunilada, o que mostra a necessidade de ajustes na equação. Na Figura 11 b observa-se a ocorrência de um padrão na disposição dos resíduos, ou seja, há a necessidade de adicionar-se um ou mais termos na equação. Assim como para a iluminância difusa, esse fato não ocorre para as condições de céu claro, como se pode observar na Figura 12b. 


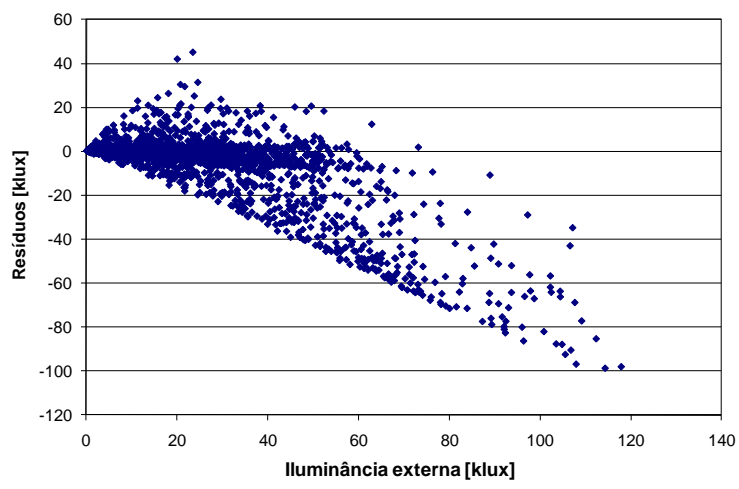

(a) Iluminância difusa medida $-1<\varepsilon \leq 1,065$

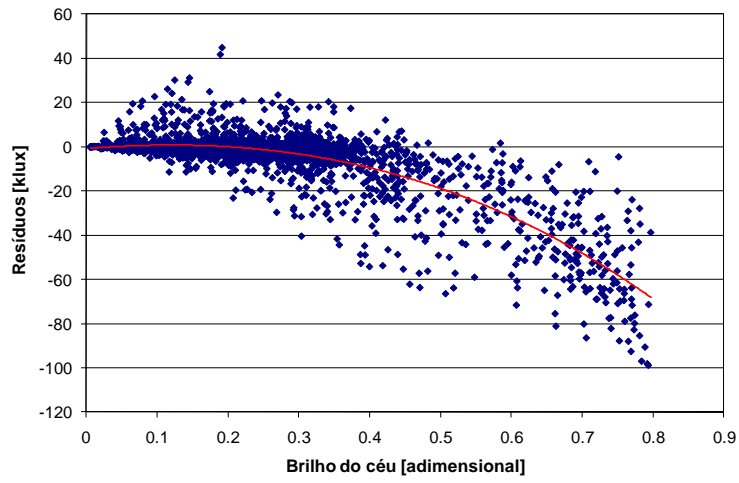

(b) Brilho do céu $-1<\varepsilon \leq 1,065$

Figura 8 - Análise dos resíduos em função dos dados estimados através do modelo de Perez et al. (1990) e do brilho do céu para céu encoberto

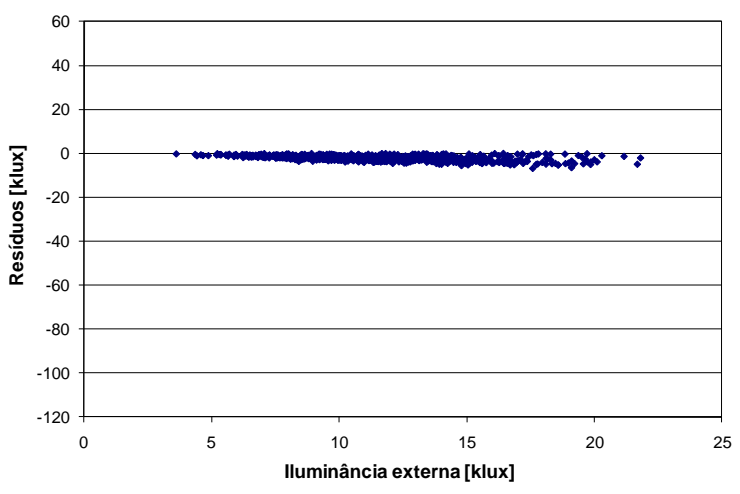

(a) Iluminância difusa medida $-\varepsilon>6,2$

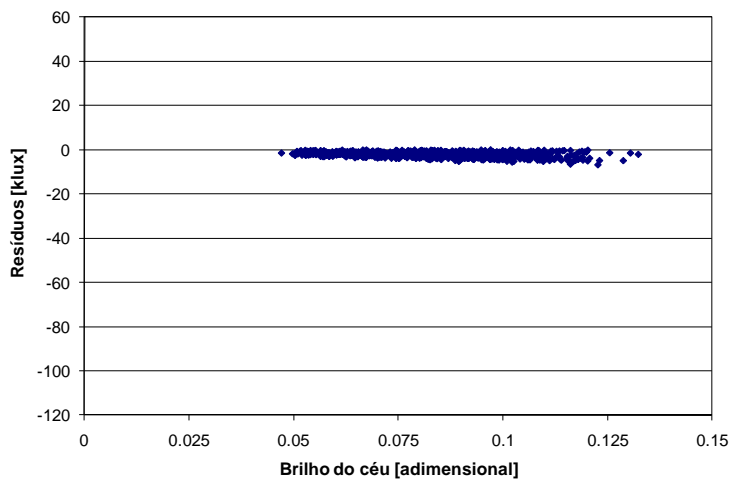

(b) Brilho do céu $-\varepsilon>6,2$

Figura 9 - Análise dos resíduos em função dos dados estimados através do modelo de Perez et al. (1990) e do brilho do céu para céu claro

\begin{tabular}{c|c|c|c}
\hline & $\begin{array}{c}\text { Iluminância Média } \\
\text { Medida [klx] }\end{array}$ & Viés [\%] & EQM [\%] \\
\hline Todos & 26,04 & 62,83 & 306,88 \\
\hline 1 & 10,60 & $-38,70$ & 161,51 \\
\hline 2 & 11,89 & 9,78 & 219,97 \\
\hline 3 & 14,54 & 21,36 & 206,77 \\
\hline 4 & 19,90 & 12,48 & 123,66 \\
\hline 5 & 28,15 & 23,19 & 131,67 \\
\hline 6 & 34,67 & 31,09 & 129,77 \\
\hline 7 & 41,21 & 4,42 & 34,00 \\
\hline 8 & 56,69 & 7,31 & 20,58 \\
\hline
\end{tabular}

Tabela 3 - Iluminância direta média medida e avaliação estatística do modelo de Perez para cada classe de céu, a partir de dados de Florianópolis de 2003 a 2005 


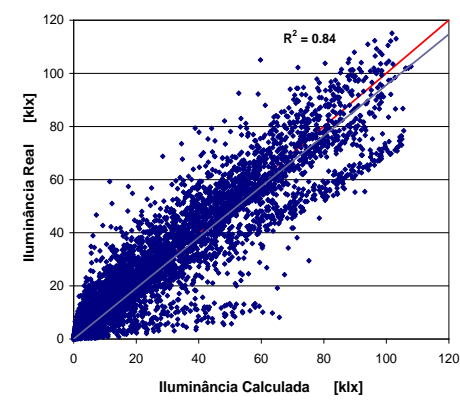

(a) Todos os dados

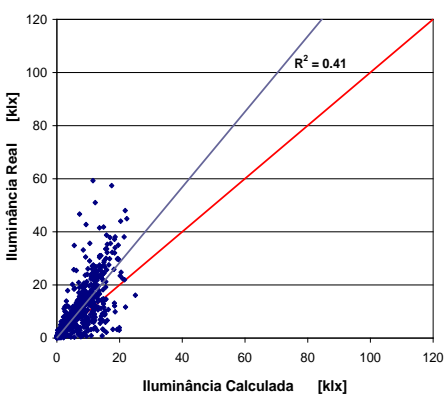

(d) $1,23<\varepsilon \leq 1,5$

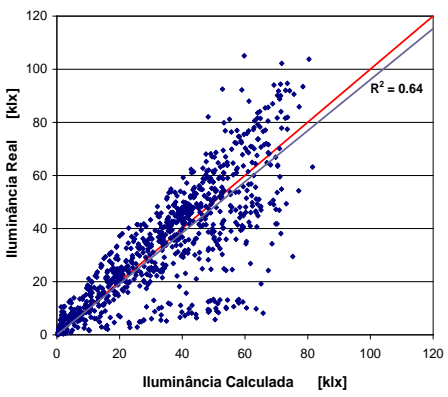

(g) $2,8<\varepsilon \leq 4,5$

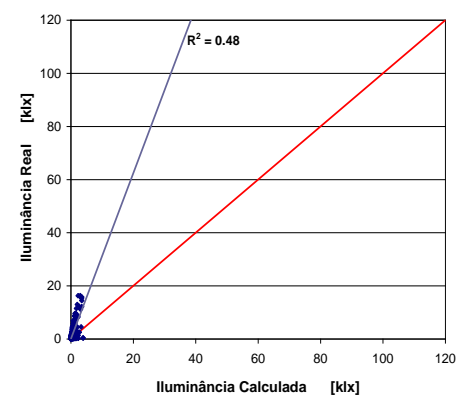

(b) Céu encoberto $-1<\varepsilon \leq$ 1,065

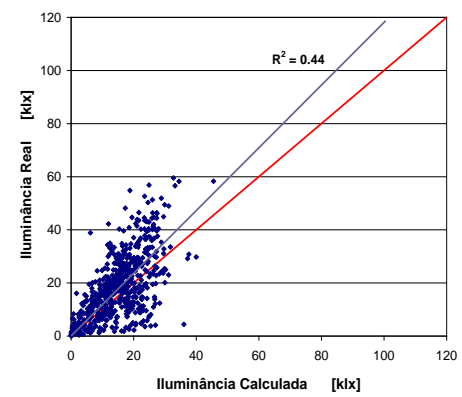

(e) $1,5<\varepsilon \leq 1,95$

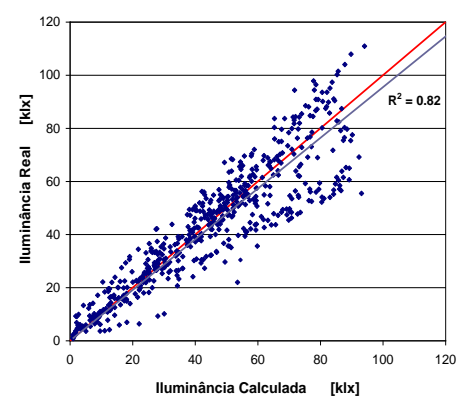

(h) $4,5<\varepsilon \leq 6,2$

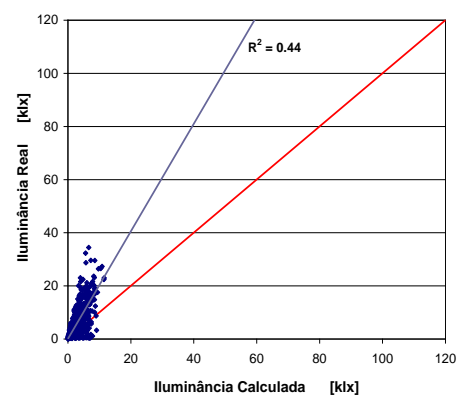

(c) $1,065<\varepsilon \leq 1,23$

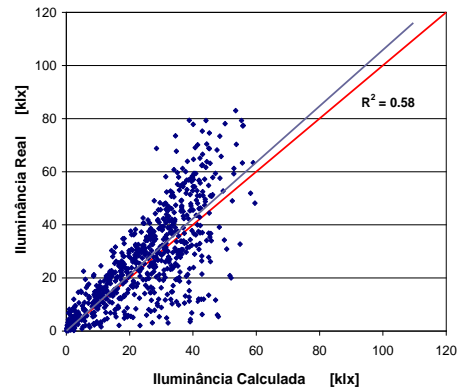

(f) $1,95<\varepsilon \leq 2,8$

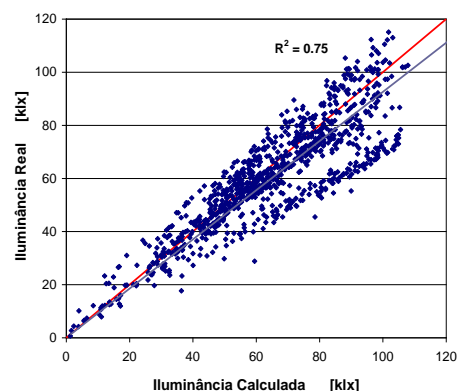

(i) Céu claro $-\varepsilon>6,2$

Figura 10 - Correlação entre as iluminâncias diretas medidas e as calculadas pelo modelo de Perez (1990), através de dados coletados em Florianópolis, entre 2003 e 2005

\section{Conclusões}

A partir da metodologia apresentada neste artigo, as simulações foram realizadas através dos programas EnergyPlus, Daysim/Radiance e TropLux. Os programas Daysim/Radiance e TropLux possuem métodos de cálculos semelhantes, mas com base em modelos de céu diferentes. Os programas EnergyPlus e Daysim/Radiance possuem métodos de cálculo diferentes, mas com base no mesmo modelo de céu.

A análise do Fator de Luz Diurna (FLD) mostra que uma das deficiências do programa EnergyPlus está no cálculo da iluminância interna. Nas salas profundas, o programa apresenta um FLD constante a partir do meio da sala, o que mostra a deficiência desse programa na resolução da refletância interna, uma vez que para esses pontos a iluminância é resultante, principalmente, da parcela de luz refletida no ambiente. Tal deficiência irá influenciar principalmente as análises realizadas quanto ao consumo de energia pelo uso da iluminação natural, referente à parcela da sala mais afastada da abertura, uma vez que os resultados da iluminância para essa parte da sala são majorados pelo programa.

Para a análise do modelo de Perez compararam-se as iluminâncias medidas com as calculadas a partir de parâmetros coletados juntamente com as iluminâncias. Nessas comparações percebeu-se que os valores calculados superestimam as iluminâncias e, quanto mais claro o céu, melhor a correlação entre as duas grandezas e menor o erro encontrado. Ou seja, tanto para a iluminação difusa quanto para a iluminância direta, os maiores erros ocorrem para as condições de céu mais encoberto. A análise dos resíduos ilustra melhor a necessidade de ajustes nos modelos de céu de Perez, principalmente para os modelos de céu encoberto, 
em que a inclusão de mais variáveis na equação pode trazer melhorias nos resultados.

Em resumo, as comparações entre as iluminâncias externas medidas e as calculadas mostram a necessidade da busca de modelos de previsão de iluminâncias que respondam de forma mais adequada às condições de céu encontradas na região estudada.

A partir das análises apresentadas neste trabalho e das conclusões acima referidas, percebe-se que o

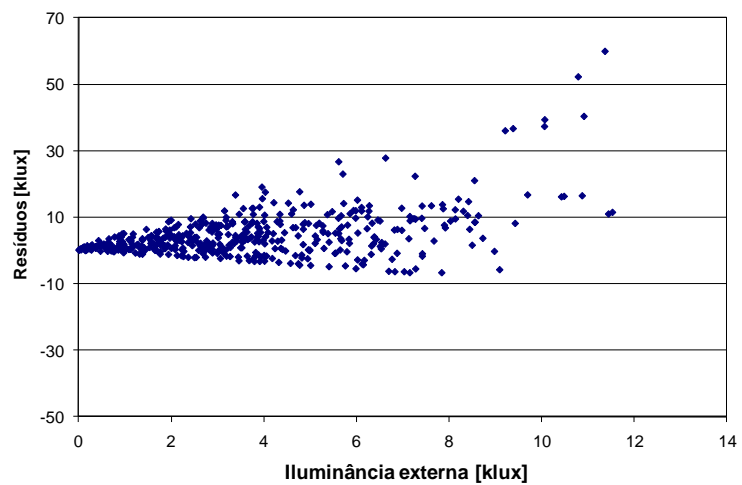

(a) Iluminância direta medida $-1,065<\varepsilon \leq 1,23$

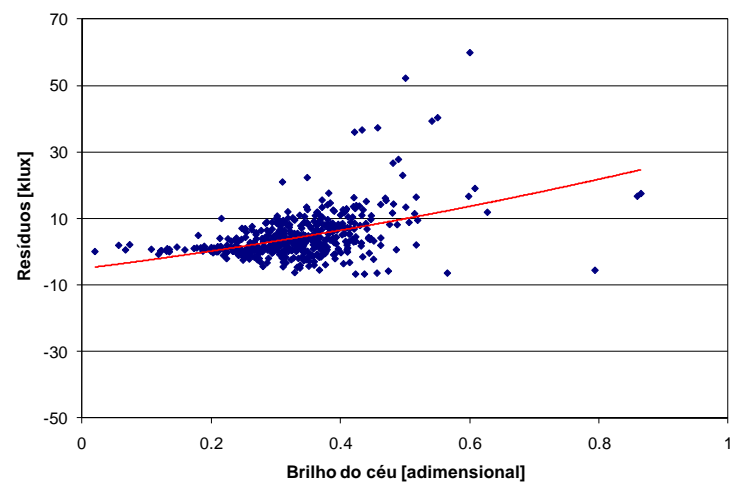

(b) Brilho do céu $-1,065<\varepsilon \leq 1,23$

Figura 11 - Análise dos resíduos em função dos dados estimados através do modelo de Perez et al. (1990) e do brilho do céu para céu encoberto

\section{Referências}

CABÚS, R. C. TropLux: um sotaque tropical na simulação da luz natural em edificações. In: ENCONTRO LATINO-AMERICANO, 4. ENCONTRO NACIONAL SOBRE CONFORTO NO AMBIENTE CONSTRUÍDO, 8., 2005, Maceió, Anais... Porto Alegre: ANTAC, 2005. 1 CD-ROM. uso do programa EnergyPlus para o cálculo das iluminâncias internas ou da economia de energia elétrica pelo uso da iluminação natural não é aconselhável para o clima analisado, pois resulta em valores bem maiores que os medidos. Os valores de iluminância elevados estão relacionados principalmente ao método simplificado de resolução da reflexão interna e aos altos valores das iluminâncias externas, calculadas através do modelo de Perez et al. (1990).

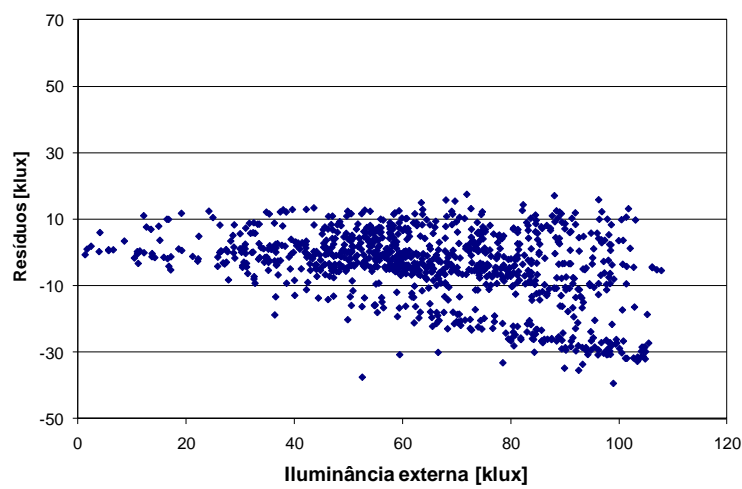

(a) Iluminância direta medida $-\varepsilon>6,2$

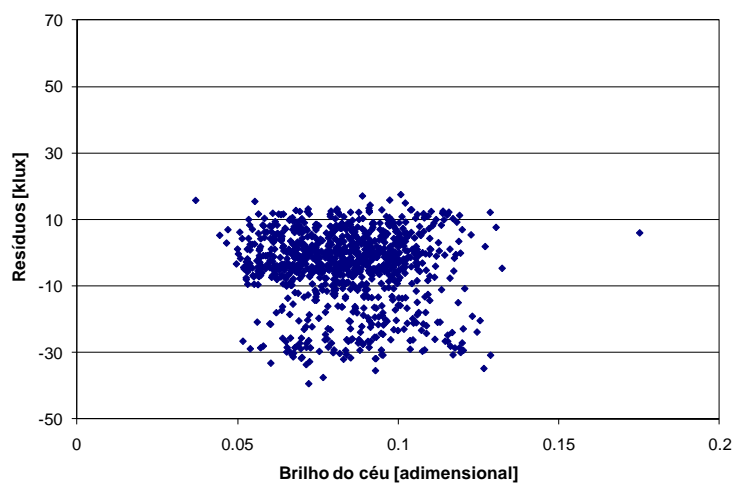

(b) Brilho do céu $-\varepsilon>6,2$

Figura 12 - Análise dos resíduos em função dos dados estimados através do modelo de Perez et al. (1990) e do brilho do céu para céu claro

CRAWLEY, D. B. et al. Energyplus: a newgeneration building energy simulation program. In: IBPSA BUILDING SIMULATION

CONFERENCE AND EXHIBITION, 6., 1999, Kyoto. Proceedings... Kyoto: IBPSA, 1999. 1 CD-ROM.

ENERGYPLUS. EnergyPlus Engineering

Reference: the reference to EnergyPlus calculations. Washington, DC: US Department of Energy, 2007.

JOHNSON, R. A.; WICHERN, D. W. Applied Multivariate Statistical Analysis. 4. ed. New Jersey: Prentice Hall, 1998. 
LOUTZENHISER, P. G.; MAXWELL, G. M.; MANZ, H. An Empirical Validation of the Daylighting Algorithms and Associated Interactions in building energy simulation programs Using Various Shading Devices and Windows. Energy, v. 32, n. 10, p. 1855-1870, Oct. 2007.

PEREZ, R. et al. Modeling Daylight Availability and Irradiance Components from Direct and Global Irradiance. Solar Energy, Kidlinton, v. 44, n. 5, p. 271-289, 1990.

RAMOS, G.; GHISI, E. Comparação de Iluminâncias Internas e Externas obtidas por meio de Simulação Computacional. In: ENCONTRO

NACIONAL DE TECNOLOGIA DO

AMBIENTE CONSTRUÍDO, 12., 2008,

Fortaleza, Anais... Porto Alegre: ANTAC, 2008.
REINHART, C. F. Tutorial on the Use of Daysim Simulations for Sustainable Design. Ottawa: Institute for Research in Construction, National Research Council Canada, 2006.

REINHART, C. F.; WALKENHORST, O. Validation of Dynamic RADIANCE-Based Daylight Simulations for a Test Office with External Blinds. Energy and Buildings, Lausanne, v. 33, n. 7, p. 683-697, Sept. 2001.

WINKELMANN, F. C.; SELKOWITZ, S. Daylighting Simulation in the DOE-2 Building Energy Analysis Program. Energy and Buildings, Lausanne, v. 8, n. 4, p. 271-286, Dec. 1985. 